\title{
Mirizzi syndrome
}

\author{
Col PV Rama Mohan*, Wg Cdr M Kumar+, Maj R Pacharu\#
}

MJAFI 2011;67:280-281

\section{INTRODUCTION}

Mirizzi syndrome (MS) is a rare form of obstructive jaundice that occurs as an infrequent complication of gallstones in about $0.1-0.7 \%$ of patients who have gallstones. ${ }^{1}$ Mirizzi was the first to describe this phenomenon as "functional hepatic syndrome" in $1948 .^{2}$ It is a benign condition resulting from a chronically impacted stone or stones in the neck of gall bladder or cystic duct, which over time induces sufficient pericholecystic inflammation to narrow and obstruct the adjacent common hepatic duct.

We present a case of MS type II who presented with obstructive jaundice.

\section{CASE REPORT}

A 50-year-old male patient, a smoker and consumer of alcohol, presented with features of painless progressive jaundice, anorexia, weight loss, and passing clay coloured stool for two months duration. Clinical examination revealed icterus, firm, non-tender hepatomegaly with rounded edge and a palpable nodule in it $8 \mathrm{~cm}$ below the right subcostal margin. Gall bladder was not palpable.

\section{EVALUATION}

\section{Liver Function Tests}

Serum bilirubin $25.3 \mathrm{mg} / \mathrm{dL}$; conjugated $17.6 \mathrm{mg} / \mathrm{dL}$; ALP $184 \mathrm{IU} /$ dL (15-112). USG abdomen showed mild central IHBR dilatation, GB contracted with sludge in its lumen and a $29 \mathrm{~mm}$ calculus at the neck. CECT abdomen showed asymmetric wall thickening of GB, without any fat stranding or liver infiltration. A $29 \mathrm{~mm}$ calculus seen in cystic duct with extrinsic compression of common hepatic duct. Distal common bile duct not visualised. MRCP showed a $3 \mathrm{~cm}$ calculus in the neck/Hartmann's pouch compressing CHD and proximal CBD (Figure 1). Cystic duct not seen.

\footnotetext{
*Senior Advisor (Surgery), MH, Jaipur, Rajasthan, ${ }^{+}$Associate Professor, \#Resident, Department of Surgery, AFMC, Pune.

Correspondence: Col PV Rama Mohan, Senior Advisor (Surgery), Military Hospital Jaipur, Rajasthan. E-mail:pvrm2007@rediffmail.com
}

Received: 15.04.2010; Accepted: 21.11.2010 doi: 10.1016/S0377-1237(11)60062-2

\section{Intra-operative}

Liver had a prominent Reidel's lobe which was palpable as a separate nodule clinically. The $3 \mathrm{~cm}$ stone in Hartmann's pouch and eroding into CHD producing a fistula (Mirizzi type 2). There was pus in the gall bladder, peri-choleductal tissues were acutely inflamed. Distal CBD found patent. Patient underwent subtotal cholecystectomy and T-tube drainage through remaining GB stump (Figure 2). Postoperative T-tube drainage was approximately $600 \mathrm{~mL} /$ day, with no features of cholangitis. As high T-tube output continued, position of T-tube confirmed with USG and T-tube cholangiogram done showed free flow of contrast into duodenum, with no evidence of leak. Patient started on bile re-feeds. Gradually, T-tube drainage reduced and T-tube was removed on the 25th postoperative day. Frozen section biopsy and histopathological examination of removed gall bladder did not reveal any evidence of malignancy. Patient reviewed six months postoperative remained asymptomatic.

\section{DISCUSSION}

Mirizzi syndrome is a rare complication of gall stones that an average general surgeon will encounter MS only a few times during his or her career. Clinical diagnosis is difficult. There are no pathognomonic features in either the history or the physical examination. No diagnostic method or clinical feature has

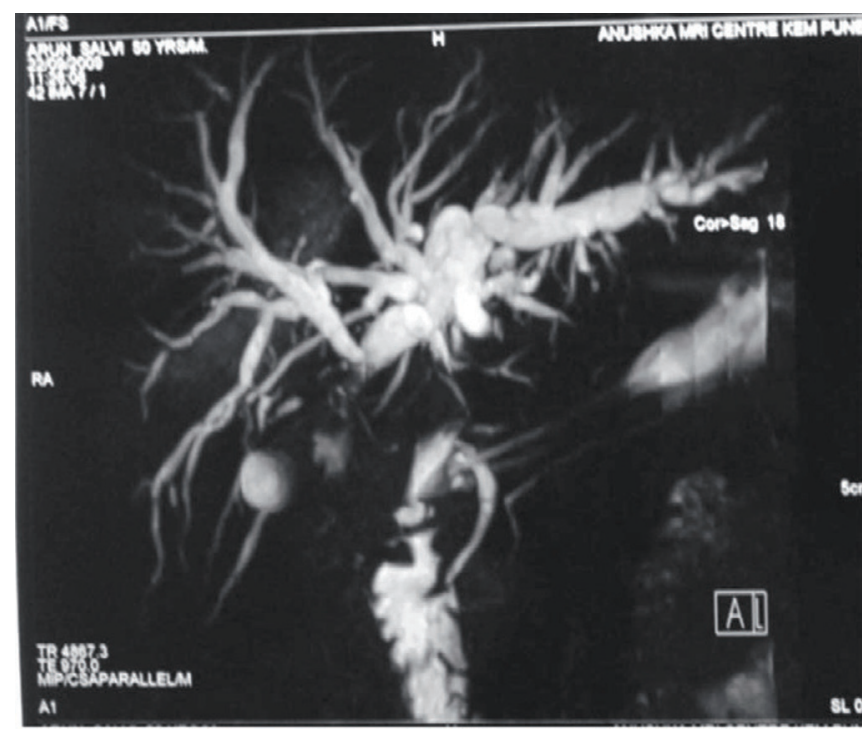

Figure 1 Magnetic resonance cholangiopancreatography picture. 


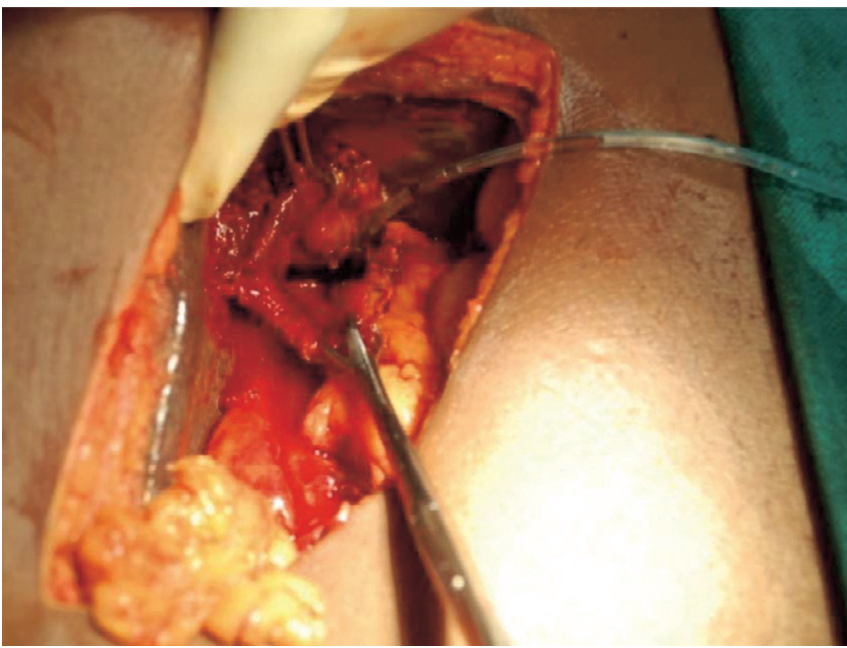

Figure 2 Insertion of a T-tube through the fistula.

$100 \%$ sensitivity and specificity. Its importance has been highlighted as a clinical entity associated with a high incidence of biliary injuries and demanding complex surgical procedures. Additionally, a high coincidence of MS and gallbladder cancer has been reported in several studies. ${ }^{3}$

In our case at initial presentation, presence of prominent Riedel's lobe gave a nodular feel to liver giving a clinical impression of secondaries liver. Despite absence of any features of acute inflammation per up gall bladder had pus in its lumen and pericholeductal tissues acutely inflamed. Presence of this inflammation made it difficult to explore CBD and confined the surgery to partial cholecystectomy, over sewing of the gallbladder cuff, and insertion of a T-tube through the fistula. Despite patent distal CBD patient continued to have high T-tube output, most probably due to inflammatory oedema of CBD forcing us to start patient on bile refeeds mixed with honey.

The condition was classified by McSherry and colleagues in 1982 and the modification of the same by Csendes and colleagues ${ }^{4}$ in 1989 into classes 1-4 is the currently accepted classification. The Csendes classification of MS is as follows:

Type 1: external compression of the common bile duct $-11 \%$.

Type 2: cholecystobiliary fistula is present involving less than one-third the circumference of the bile duct - $41 \%$.

Type 3: a fistula is present involving upto two-third the circumference of the bile duct $-44 \%$.

Type 4: a fistula is present with complete destruction of the wall of the bile duct $-4 \%$.

Management of MS in cases in whom the bile duct is inflamed and no fistula is present (Type I), patients can be managed with cholecystectomy. Many surgeons contend that laparoscopic cholecystectomy is contraindicated in $\mathrm{MS}^{5}$ while the others consider the laparoscopic technique feasible, though technically demanding. ${ }^{6}$ The common hepatic duct almost always returns to normal after the offending stone has been removed by cholecystectomy and the inflammatory process has resolved. Rarely a well-established stricture presents months to years after the acute episode. In such cases, stricture repair of the distal bile duct is indicated for persistent strictures using either Roux-en-Y choledochojejunostomy or choledochoduodenostomy.

If cholecystocholedochal fistula is present, partial cholecystectomy, oversewing the gallbladder cuff, and insertion of a T-tube through the fistula as adequate treatment for Type 2 disease. ${ }^{7}$ Baer et $\mathrm{al}^{8}$ suggested the placement of a T-tube through a separate choledochotomy in the distal CBD in order to prevent excessive leakage and stricture at the fistula site. The presence of CBD stones, fistula, or stenosis at the site of impaction of the stone may necessitate CBD exploration and/or the insertion of a T-tube. Choledochoduodenostomy for Type 3 disease, and biliary bypass (Roux-en-Y hepaticojejunostomy) for Type 4 MS are the treatment of choices. In all cases, a frozen section of the gallbladder wall should be done to rule out coexistent cancer.

The prognosis of MS is very good for type 1 lesions, as simple cholecystectomy is all that is necessary for cure. In treating more serious types with fistulous destruction of the common duct, postoperative morbidity rises, with $10 \%$ or more biliary fistulae, biliary stricturing requiring dilation or reoperation, or hepatic abscesses requiring drainage. ${ }^{9}$

\section{REFERENCES}

1. Hazzan D, Golijanin D, Reissman P. Combined endoscopic and surgical management of Mirizzi syndrome. Surg Endosc 1999;13: 618-620.

2. Mirizzi PL. Sindrome del conducto hepatico. J Int Chir 1948;8: 731-733.

3. Prasad TL, Kumar A, Sikora SS, Saxena R, Kapoor VK. Mirizzi syndrome and gallbladder cancer. J Hepatobiliary Pancreat Surg 2006; 13:323-326.

4. Csendes A, Diaz JC, Burdiles P, Maluenda F, Nava O. Mirizzi syndrome and cholecystobiliary fistula: a unifying classification. Br J Surg 1989;76:1139-1143.

5. Moser JJ, Baer HU, Glatti A. Mirizzi syndrome eda contraindication for laparoscopic surgery. Helv Chir Acta 1993;59:577-580.

6. Meng WC, Kwok SP, Kelly SB, Lau WY, Li AK. Management of Mirizzi syndrome by laparoscopic cholecystectomy and laparoscopic ultrasonography. BrJ Surg 1995;82:396.

7. Corlette $\mathrm{MB}$, Bismuth $\mathrm{H}$. Biliobiliary fistula. A trap in the surgery of cholelithiasis. Arch Surg 1975;110:377-383.

8. Baer HU, Matthews JB, Schweizer WP, Gertsch P, Blumgart LH. Management of the Mirizzi syndrome and the surgical implications of cholecystcholedochal fistula. Br J Surg 1990;77:743-745.

9. Waisberg J, Corona A, Abreu I. Benign obstruction of the common hepatic duct (Mirizzi syndrome): diagnosis and operative management. Arq Gastroenterol 2005;42:13-18. 